\title{
A Comparison of Cerebral SPECT Abnormalities in HIV-Positive Homosexual Men With and Without Cognitive Impairment
}

Ned Sacktor, MD; Ronald L. Van Heertum, MD; George Dooneief, MD, MPH; Jack Gorman, MD; Alexander Khandji, MD; Karen Marder, MD, MPH; Ramy Nour, MD; George Todak, MSW; Yaakov Stern, PhD; Richard Mayeux, MD, MSE

\begin{abstract}
Objective: To determine whether technetium Tc $99 \mathrm{~m}$ exametazime (HMPAO) single-photon emission computed tomography (SPECT) can distinguish between human immunodeficiency virus (HIV)-positive homosexual men with normal neuropsychologic test results and HIV-positive homosexual men with abnormal neuropsychologic test results.
\end{abstract}

Design: Neurologic, neuropsychologic, magnetic resonance imaging, and Tc 99m HMPAO SPECT examinations were performed on $10 \mathrm{HIV}$-positive homosexual men without cognitive impairment and five HIV-positive homosexual men with cognitive impairment.

Patients: Human immunodeficiency virus-positive homosexual men from New York City were recruited for the study.

Main Outcome Measures: Findings on SPECT scans were evaluated qualitatively for focal defects, heterogeneity of the cortical margin, white matter hypoperfusion, and decreased global cortical uptake. All SPECT focal defects were coregistered with magnetic resonance images; SPECT heterogeneity and global cortical uptake were also measured quantitatively.

Resulis: Coregistration with magnetic resonance imaging revealed that $63 \%$ of the focal SPECT defects corresponded to brain gyri and $37 \%$ corresponded to sulci. There was no significant difference in the frequency of qualitative or quantitative SPECT abnormalities between HIV-positive homosexual men with and without cognitive impairment. However, after examining individual neuropsychologic test factors, impaired motor speed performance was associated with decreased quantitative global cerebral uptake.

Conclusions: Qualitative SPECT abnormalities are not increased in frequency in HIV-positive homosexual men with global cognitive impairment compared with those in HIV-positive homosexual men without cognitive impairment. Impaired motor speed performance may be associated with decreased quantitative global cerebral uptake.

(Arch Neurol. 1995;52:1170-1173)
From the Gertrude $\mathrm{H}$.

Sergievsky Center

(Drs Sacktor, Dooneief,

Marder, Stern, and Mayeux); the HIV Center for Clinical and Behavioral Studies, New York State Psychiatric Institute (Drs Sacktor, Dooneief, Gorman, Marder, Stern, and Mayeux and Mr Todak); and the

Departments of Neurology (Drs Sacktor, Dooneief, Marder, Stern, and Mayeux), Radiology (Drs Van Heertum, Khandji, and Nour), and Psychiatry (Drs Gorman, Stern, and Mayeux and Mr Todak), Columbia University College of Physicians and Surgeons, New York, NY. Dr Sacktor is currently with the Johns Hopkins Bayview Medical Center, Baltimore, $M d$.

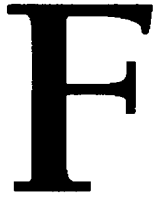
(HIV) type 1-infected homosexual men without a history of parenteral drug use may demonstrate focal cortical or subcortical perfusion defects, a heterogeneous pattern of cortical uptake, periventricular white matter hypoperfusion, and decreased global cortical uptake. ${ }^{1-10}$ Abnormal findings on SPECT scans have been reported in HIV-positive subjects both with and without cognitive impairment. ${ }^{11}$ The current study was designed to evaluate whether abnormalities depicted on technetium Tc $99 \mathrm{~m}$ exametazime (HMPAO) SPECT could distinguish between HIV-positive homosexual men with mild cognitive impairment and
HIV-positive homosexual men without cognitive impairment.

\section{RISSUITS}

Ten subjects were without cognitive impairment, and five subjects (three subjects with HIV-1-associated minor cognitive and/or motor disorder, two subjects with HIV-1-associated mild dementia complex) had cognitive impairment (Table). For the five subjects with cognitive impairment, abnormal cognitive domains were memory (the Selective Reminding Test $[n=3])$, verbal fluency

\section{See Subjects and Methods} on next page 


\section{SUBJECTS AND METHODS}

\section{SUBJECTS}

Fifteen subjects were recruited from a cohort of homosexual volunteers enrolled in a study of HIV infection described previously. ${ }^{11-13}$ Subjects who had a history of depression, psychosis, or cocaine or crack use were excluded.

\section{METHODS}

\section{Medical, Neurologic, and}

Neuropsychologic Evaluations

Subjects underwent a structured neurologic examination, including history, by a neurologist (N.S.), CD4 lymphocyte count, and neuropsychologic tests, as described elsewhere. ${ }^{12}$ Cognitive domains included memory, language, frontal (or executive) function, attention, visuospatial function, and motor speed. Cognitive impairment was defined as abnormal if there was defective performance on one test or borderline performance on two tests. The diagnosis of cognitive impairment was made by investigators who were blinded to the results of the SPECT images. Individual neuropsychologic test factors were also examined. Motor speed was represented by the grooved pegboard time (pegs with dominant hand) ${ }^{14}$; memory, by Selective Reminding Test total score ${ }^{15}$; language, by number of words reported on the Controlled Oral Word Association test ${ }^{16}$; frontal (or executive) function, by Odd Man Out total score ${ }^{17}$; and attention, by Digit Symbol age-scaled score..$^{18,19}$

\section{Magnetic Resonance Imaging Scans}

Magnetic resonance imaging (MRI) scans were performed on a 1.5-T system (Signa, General Electric, Milwaukee, Wis). The imaging protocol consisted of $T_{1}$-weighted (repetition time $[T R]=500$ and echo time $[T E]=10), T_{2}$-weighted (TR=3500 and $T E=85$ ), and heavily $T_{2}$-weighted ( $T R=7100$ and $T E=288$ ) axial and coronal scans. ${ }^{11}$ Magnetic resonance imaging scans were evaluated for atrophy, small areas of high signal intensity, and larger focal lesions by one neuroradiologist (A.K.).

\section{SPECT Scans}

Subjects were injected with $82 \times 10^{10} \mathrm{mBq}$ of technetium Tc $99 \mathrm{~m}$ exametazime (Ceretec, Amersham Ltd, Amersham, England). Acquisition was performed on a triplehead SPECT gamma camera (Prism-3000, Picker, Cleveland, Ohio), with a resolution of $7.8-\mathrm{mm}$ full-width half maximum, using low-energy, ultra-high resolution fan beam collimation. Acquisition consisted of a multiple rapid acquisition sequence technique, with a radius of rotation of less than $14 \mathrm{~cm}$. After a Butterworth filter (order, 5 ; cutoff, 0.31 to 0.35 ) was applied, attenuation connection and ramp filter back-projection were performed.

The SPECT images were assessed qualitatively for degree of cortical heterogeneity, focal defects, global cortical uptake, and diminished white matter uptake. Focal cortical defects were qualitatively defined on the basis of visually observable side-to-side asymmetry in the transaxial plane images with confirmatory changes evident in at least one additional image plane. A SPECT scan with more than one focal defect was defined as abnormal. Cortical heterogeneity was classified as either normal or abnormal. Global cortical uptake was estimated qualitatively to be either normal or reduced in comparison with observed tracer uptake in the cerebellum. White matter uptake was estimated without knowledge of ventricular size as normal or abnormal. Any degree of severity (mild, moderate, or severe) of heterogeneity, decreased global cortical uptake, or white matter uptake was defined as abnormal. A quantitative estimation of heterogeneity was also performed with a standardized region of interest (ROI) template, 6 pixels (13.8 $\mathrm{mm}$ ) in width, encompassing the entire cortex at the level of the thalami. The cortical counts (mean \pm SD) were obtained within the ROI, and a coefficient of variation analysis was calculated. In addition, mean counts in the whole cortical ROI were determined and normalized to mean cerebellar cortical ROI counts (quantitative global uptake).

Focal defects were also evaluated using crosssectional horizontal profile analysis to quantify the degree of side-to-side asymmetry. Asymmetry of greater than $15 \%$ was considered significant. Transaxial SPECT and MRI images were coregistered and fused to further assess each focal defect confirmed by the horizontal profile analysis. The appropriate SPECT sections were superimposed on the corresponding $T_{2}$-weighted MRI images using a standard software package (Adobe Photoshop, Macintosh version 2.5.1; Adobe Systems Inc, Mountain View, Calif).

\section{Data Analysis}

Neuropsychologic, MRI, and qualitative SPECT data were each collapsed into dichotomous variables (normal and abnormal). Group comparisons were made between HIVpositive subjects with and without cognitive impairment, using Student's $t$ tests for quantitative data and Fisher's exact test (two tailed) to compare proportions. A linear regression model, controlling for age and education, was used to determine whether quantitative heterogeneity or global cortical uptake was associated with cognitive impairment or with an abnormal neuropsychologic test factor.
(Animal Naming ${ }^{20}[\mathrm{n}=4]$ and Controlled Oral Word Association test $(n=2])$, attention (Digit Symbol test from the Wechsler Adult Intelligence Scale battery $[n=1]$ ), and executive function (Odd Man Out $[\mathrm{n}=1]$ ). Subjects with cognitive impairment were older and had a lower CD4 lymphocyte count. There was no significant difference in atrophy or the presence of white matter hyperintensities on MRI scans between the two groups.

Focal defects depicted on SPECT scans were seen in all cortical areas as well as the basal ganglia and thala- mus. After coregistration, $63 \%$ of the defects corresponded to cortical gyri ("true" defects), whereas $37 \%$ corresponded to sulci. One of the sulcal defects, a posterior frontal perisylvian defect, corresponded to focal atrophy on MRI. One subject with focal neurologic signs (increased deep tendon reflexes on the left side) had a true right frontal focal defect depicted on SPECT scan.

There were no differences in the presence of gyral SPECT focal defects, cortical heterogeneity (qualitative or quantitative), white matter hypoperfusion, or global 


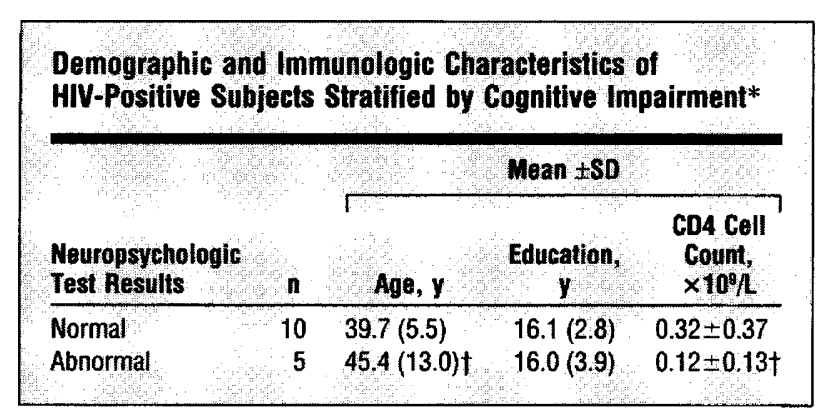

*HIV indicates human immunodeficiency virus.

$\dagger P<.05$.

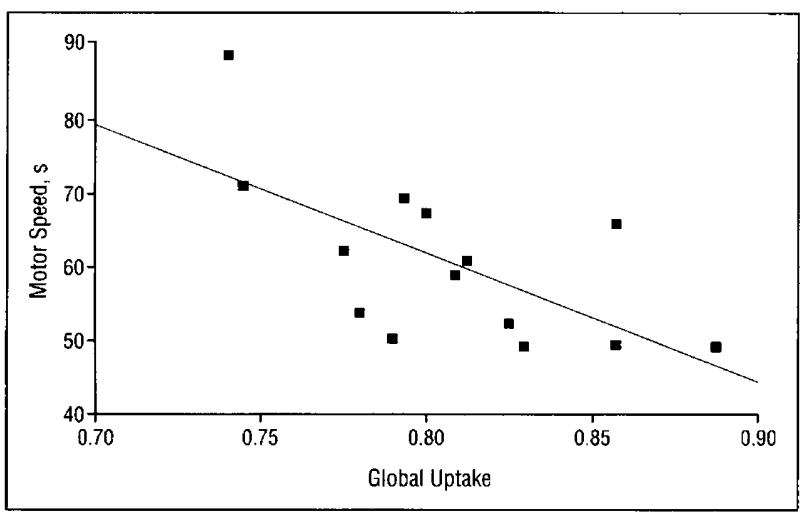

Regression line of quantitative global uptake as predicted by motor speed test performance (grooved pegboard time in seconds using pegs with dominant hand); adjusted for age and education.

cortical uptake (qualitative or quantitative) between subjects with and without cognitive impairment. However, the four subjects with the least heterogeneity (measured quantitatively) had normal neuropsychologic test results. The three subjects with the lowest global cortical uptake (measured quantitatively) had cognitive impairment.

A linear regression model was used to examine the association of quantitative global cortical uptake or heterogeneity and specific neuropsychologic factors. After controlling for age and education, decreased global uptake predicted slower motor speed test performance $(P<.03)$ ( Figure), but not memory, language, frontal, or attention performance. Heterogeneity did not predict abnormal motor speed performance, memory, language, frontal (or executive) function, or attention.

\section{COMMENT}

This study evaluated whether SPECT abnormalities are more frequent in HIV-positive homosexual men with cognitive impairment than in HIV-positive homosexual men without cognitive impairment. Similar to those obtained in HIV-l-associated dementia complex, ${ }^{21}$ after coregistration, $63 \%$ of the defects corresponded to brain gyri whereas $37 \%$ corresponded to cortical sulci. Perfusion defects corresponding to cortical sulci may represent the normal anatomy of the brain, or the sulci may be enlarged secondary to focal atrophy because of HIV infection. Only one of the sulcal defects was interpreted as focal atrophy on MRI, suggesting that the majority of false defects are brain sulci.

The current study suggests that there is no significant difference in the frequency of SPECT focal defects, cortical heterogeneity (by qualitative or quantitative evaluation), periventricular white matter hypoperfusion, or global cerebral uptake (by qualitative or quantitative evaluation) between HIV-positive homosexual men without cognitive impairment and HIV-positive homosexual men with cognitive impairment. After adjusting for age and education, decreased global uptake measured quantitatively was associated with impaired motor speed. Abnormal motor speed performance is often a sensitive indicator of cognitive impairment in HIV infection. However, the regression may be highly influenced by one subject with low global uptake and very slow motor speed; therefore, further studies to evaluate the association of motor speed performance and quantitative SPECT evaluations should be performed.

The lack of an association between SPECT abnormalities and the presence of global cognitive impairment suggests that SPECT abnormalities in HIV-positive homosexual men may precede the development of cognitive impairment. Another possible explanation is that the pathogenesis of the SPECT abnormalities and the abnormalities on neuropsychologic testing may be different. Pathologically, multinucleated giant cells and diffuse myelin pallor are specific for HIV-associated dementia, but these changes occur in only $50 \%$ of the patients with dementia. ${ }^{22}$ Indirect mechanisms of brain dysfunction, such as abnormal cytokine expression, may also contribute to the pathogenesis of HIV-associated dementia. In asymptomatic HIV-infected individuals, an inflammatory reaction consisting of leptomeningitis and vasculitis may be seen. ${ }^{23,24}$ This vascular inflammation may be responsible for myelin pallor and gliosis of the deep white matter. The SPECT abnormalities in both HIV-positive homosexual men with and without cognitive impairment may be a neuroimaging marker of this vascular inflammation. Cerebrospinal fluid analysis and pathologic correlations in HIVpositive subjects with a history of abnormal SPECT results would be necessary to confirm this hypothesis. An alternative hypothesis is that the SPECT abnormalities may reflect abnormal brain function that is unrelated to direct vascular injury.

Compared with those in HIV-positive homosexual men without cognitive impairment, qualitative SPECT abnormalities are not increased in frequency in HIVpositive homosexual men with mild global cognitive impairment. Impaired motor speed performance may be associated with decreased quantitative global cerebral uptake. The SPECT abnormalities may represent a neuroimaging marker that is independent of cognitive impairment in HIV infection.

Accepted for publication July 20, 1995.

This study was supported by Aaron Diamond Foundation project 817-5332C-7001 and by federal grants NIMH P50-MH43520 and MH44959.

Reprint requests to G. H. Sergievsky Center, Columbia University, $630 \mathrm{~W}$ 168th St, New York, NY 10032 (Dr Mayeux). 


\section{REFERENCES}

1. Ell P, Costa D, Harrison M. Imaging cerebral damage in HIV infection. Lancet. 1987:11:569-570.

2. Pohl P, Vogl G, Fill H, Rossler H, Zangerle R, Gerstenbrand F. Single photon emission computed tomography in AIDS dementia complex. J Nucl Med. 1988; 29:1382-1386.

3. Tatsch K, Schielke E, Bauer WM, Mark| A, Einhaupl K, Kirsch C. Functional and morphological findings in early and advanced stages of HIV infection: a comparison of $99 \mathrm{mTC}-\mathrm{HMPAO}$ SPECT with CT and MRI studies. Nuklearmedizin. 1990;29:252-258.

4. Tran Dinh Y, Mamo H, Cervoni J, Caulin C, Saimot A. Disturbances in the cerebral perfusion of human immune deficiency virus-1 seropositive asymptomatic subjects: a quantitative tomography study of 18 cases. J Nucl Med. 1990; $31: 1601-1607$.

5. Masdeu JC, Yudd A, Van Heertum RL, et al. Single-photon emission computed tomography in human immunodeficiency virus encephalopathy: a preliminary report. J Nucl Med. 1991;32:1471-1475.

6. Van Heertum RL, Miller SH, Mosesson RE. SPECT brain imaging in neurologic disease. Radiol Clin North Am. 1993;31:881-907.

7. Woods $S$, O'Malley $S$, Martini B, et al. SPECT regional cerebral blood flow and neuropsychological testing in non-demented HIV-positive drug abusers: preliminary results. Prog Neuro-psychopharmacol Biol Psychiatry. 1991;15:649-662.

8. Rosci M, Pigorini F, Berabei A, et al. Methods for detecting early signs of AIDS dementia complex in asymptomatic HIV-1-infected subjects. AIDS. 1992;6: 1309-1316.

9. Pau F, Rosci M, Bernabi A, et al. 99m Tc-HMPAO SPECT in asymptomatic HIV-1 infected patients: preliminary results. J Nucl Med All Sci. 1990;34:213-214.

10. Holman BL, Garada B, Johnson KA, et al. A comparison of brain perfusion SPECT in cocaine abuse and AIDS dementia complex. J Nucl Med. 1992;33: $1312-1315$.

11. Sacktor N, Prohovnik I, Van Heertum R, et al. Cerebral single-photon emission computed tomography abnormalities in human immunodeficiency virus type 1-infected gay men without cognitive impairment. Arch Neurol. 1995;52: 607-611.

12. Stern $Y$, Marder $K$, Bell $K$, et al. Multidisciplinary baseline assessment of gay men with and without HIV infection, III: neurological and neuropsychological findings. Arch Gen Psychiatry. 1991;48:131-138.

13. Gorman J, Kertzner R, Todak G, et al. Multidisciplinary baseline assessment of gay men with and without HIV infection, I: overview of study design. Arch Gen Psychiatry. 1991;48:120-123.

14. Tiffin J. Perdue Pegboard: Examiner Manual. Chicago, III: Science Research Association; 1968.

15. Buschke $H$, Fuld PA. Evaluating storage, retention, and retrieval in disordered memory and learning. Neurology. 1974;24:1019-1025.

16. Benton A. FAS test. In: Spreen 0, Benton A, eds. Neurosensory Center Comprehensive Examination for Aphasia. Victoria, British Columbia: University of Victoria; 1967.

17. Flowers K, Robertson C. The effect of Parkinson's disease on the ability to maintain a mental set. $J$ Neurol Neurosurg Psychiatry. 1985;48:517-529.

18. Wechsler D. Wechsler Adult Intelligence Scale-Revised. New York, NY: The Psychological Corp; 1981

19. Stern $Y$, Liu X, Marder $K$, et al. Neuropsychological changes in a prospectively followed cohort of gay and bisexual men with and without HIV. Neurology. 1995;45:467-472

20. Goodglass H, Kaplan D. The Assessment of Aphasia and Related Disorders 2nd ed. Philadelphia, Pa: Lea \& Febiger; 1983.

21. Johnson K, Becker J, Gerada B, et al. Superimposition of SPECT/MR images in AIDS dementia complex. Neurology. 1993;43:A187.

22. Glass $\mathrm{J}$, Wesselingh $S$, Selnes 0 , et al. Clinical-neuropathologic correlation in HIV-associated dementia. Neurology. 1993;43:2230-2237.

23. Gray $F$, Hurtrel M, Hurtrel B. Early central nervous system changes in human immunodeficiency virus (HIV)-infection. Neuropathol Appl Neurobiol. 1993;19: 3-9.

24. Gray F, Lescs M-C, Paraire F, et al. Early brain changes in HIV infection: neuropathological study of 11 HIV seropositive, non-AIDS cases. J Neuropathol Exp Neurol. 1992:51:177-185.

\section{YOUR ACCESS TO THE WORLD OF MEDICINE}

- Abstracts, tables of contents and medical news briefs

- Weekly science news releases

- Current career opportunities
- Full text of Archives Journal Club/ Women's Health

- Links to other medical resources

- More features coming soon! 